\title{
Line edge roughness metrology software
}

Cite as: J. Vac. Sci. Technol. B 38, 012602 (2020); https://doi.org/10.1116/1.5122675

Submitted: 30 July 2019 . Accepted: 13 December 2019 . Published Online: 26 December 2019

Sertac Guneri Yazgi, Tzvetan Ivanov, Mathias Holz, Ivo W. Rangelow, and (i) Burhanettin Erdem Alaca

\section{COLLECTIONS}

Paper published as part of the special topic on Conference Collection: The 63rd International Conference on Electron, Ion, and Photon Beam Technology and Nanofabrication (EIPBN 2019) EIPBN2019
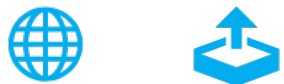

\section{ARTICLES YOU MAY BE INTERESTED IN}

Tip-based electron beam induced deposition using active cantilevers

Journal of Vacuum Science \& Technology B 37, 061812 (2019); https://doi.org/10.1116/1.5123287

Application of a B-spline model dielectric function to infrared spectroscopic ellipsometry data analysis

Journal of Vacuum Science \& Technology B 38, 014001 (2020); https://doi.org/10.1116/1.5126110

Effect of oxygen plasma cleaning on nonswitching pseudo-Bosch etching of high aspect ratio silicon pillars

Journal of Vacuum Science \& Technology B 38, 012804 (2020); https://

doi.org/10.1116/1.5122822

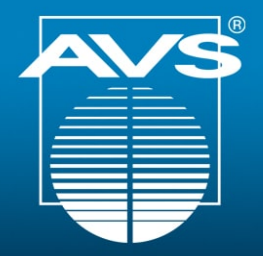

\section{Advance your science and} career as a member of

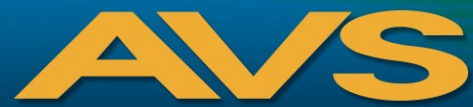




\title{
Line edge roughness metrology software
}

Cite as: J. Vac. Sci. Technol. B 38, 012602 (2020); doi: $10.1116 / 1.5122675$

Submitted: 30 July 2019 . Accepted: 13 December 2019.

Published Online: 26 December 2019

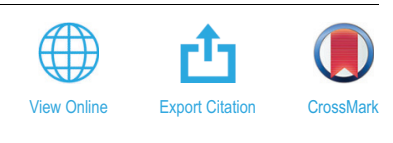

Sertac Guneri Yazgi, ${ }^{1}$ Tzvetan Ivanov, ${ }^{2}$ Mathias Holz, ${ }^{3}$ Ivo W. Rangelow, ${ }^{2}$ (D) and Burhanettin Erdem Alaca ${ }^{1,4, a)}$ (iD

\section{AFFILIATIONS}

${ }^{1}$ Department of Mechanical Engineering, Koc University, Sarıyer, 34450 Istanbul, Turkey

${ }^{2}$ Department of Micro- and Nanoelectronic Systems, TU IImenau, 98693 IImenau, Germany

${ }^{3}$ nano analytik $\mathrm{GmbH}, 98693$ IImenau, Germany

${ }^{4}$ Koc University Surface Technologies Research Center (KUYTAM), Koc University, Sarıyer, 34450 Istanbul, Turkey

Note: This paper is part of the Conference Collection: The 63rd International Conference on Electron, Ion, and Photon Beam Technology and Nanofabrication (EIPBN 2019).

${ }^{a)}$ Electronic mail: ealaca@ku.edu.tr

\begin{abstract}
A line edge roughness analysis software is developed based on the Canny edge detection algorithm with a double threshold, where threshold values are obtained by Otsu's method. The performance of the software is demonstrated on features with a 200-nm nominal pitch generated by current-controlled, field-emission scanning probe lithography. Two lithographic modes are applied: (a) direct self-development positive mode and (b) image reversal mode. Atomic force imaging is used to analyze the line edge roughness. This is followed by a benchmarking study, where findings are compared to those provided by METROLER software (Fractilia, LLC). This work is the first report on both line edge roughness involving imaging using the same exposure setup and latent image line edge roughness-made possible thanks to the resolving power of imaging through noncontact AFM. The authors are presenting a comparison of patterning through image reversal of the calixarene molecular glass resist from negative-tone to positive-tone as well as direct-write. In image reversal, a close match was observed between the proposed analysis and METROLER software for line edge roughness and linewidth.
\end{abstract}

Published under license by AVS. https://doi.org/10.1116/1.5122675

\section{INTRODUCTION}

For more than 50 years, Moore's law was followed closely while scaling down the feature size. ${ }^{1}$ With Moore's law having reached its physical limits, maintaining the miniaturization trends in the singledigit nanometer regime with a high yield and cost-effectiveness became a challenge. ${ }^{2}$ According to the International Roadmap for Devices and Systems (IRDSs), feature sizes are expected to decrease

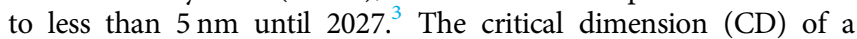
feature is the most important parameter with decreasing feature size. ${ }^{4}$ In addition to $\mathrm{CD}$, meeting the line edge roughness (LER) and linewidth roughness (LWR) requirements are the other main challenges. ${ }^{4}$ IRDS requires $1.2 \mathrm{~nm}$ metal LWR for the $5-\mathrm{nm}$ node as of 2021. ${ }^{4}$ However, currently there is no available solution to fulfill this roughness analysis requirement, posing a serious problem against further scaling of the feature size and improving the performance of the metrology technique.

With the investigation of LER as a new and ever-developing research field, there are a few commercial solutions to analyze LER and related parameters, ${ }^{5,6}$ among which METROLER software
(Fractilia, LLC) utilizes the patented Fractilia Inverse Linescan Model edge detection algorithm to measure and remove the noise without filtering to find unbiased roughness values. ${ }^{7}$ Within this study, we developed our own software implementing the Canny edge detection algorithm with a double threshold, where threshold values are obtained by Otsu's method ${ }^{8}$ within the MATLAB environment (R2015a, The MathWorks, Natick, MA) to measure edge variations. The developed software is further verified by benchmarking via METROLER software (Fractilia, LLC).

Scanning probe lithography (SPL) represents an unconventional, cost effective, and promising solution to pattern features in the single-digit regime. 9 SPL operates under controlled environmental conditions without any need for vacuum or any special gas decreasing the tool overhead, cost, and facility needs. Furthermore, the same exposure setup and tip can be used to image patterns right after exposure. ${ }^{10}$ There are a series of SPL technologies based on different working principles, including thermal/thermomechanical, bias-induced, oxidation, mechanical, and dip-pen SPL. ${ }^{11}$ Unlike electron microscopy, the imaging process is nondestructive 
and does not cause any unintentional patterning. SPL tools can be employed to pattern many types of substrates including polymers and ceramics. ${ }^{11}$

Field-emission SPL (FE-SPL) is a bias-induced technique based on Fowler-Nordheim electron emission from a very sharp tip. ${ }^{12}$ For FE-SPL, neither complex electronics nor optics are required. ${ }^{13}$ Compared to electron-beam lithography (EBL), the proximity effect is significantly reduced due to the use of ultralow-energy electrons. Although the limited throughput of scanning probe technology can be considered as a limitation for its practical applications, mix-and-match patterning can be a solution in this aspect. ${ }^{14}$ Furthermore, SPL can be used for fabricating molds for nanoimprint lithography reducing further the limitation of serial processing. ${ }^{1.5}$

After SPL patterning, a solubility change is observed in the exposed area resulting either in positive-tone patterning, if the exposed region becomes soluble, or in negative-tone patterning otherwise. ${ }^{16}$ When the resist is exposed in negative-tone patterning, a latent image is observed on the resist before development. The calixarene molecular glass resist can be used as a negative-tone resist in EBL (Ref. 17) and as a positive-tone resist in FE-SPL. ${ }^{18}$ Although negative-tone patterning in FE-SPL requires a subsequent wet development step, this is not the case for positive patterning, where the exposed area is ablated. Furthermore, the tone of the image can be altered by image reversal to benefit from the advantages of a resist of interest.

Such process variations also give rise to a new set of challenges requiring an in-depth LER analysis. LER for thermal probe maskless lithography was investigated previously through SEM micrographs. ${ }^{19}$ However, there are no previous LER reports involving imaging using the same SPL setup. Similarly, while LER of latent images was studied before in a series of papers, ${ }^{20-22}$ there was no previous report on LER for latent images in SPL. Addressing these points missing in the literature, this work investigates LER in FE-SPL for the three cases (i) latent image of negative-tone exposure, (ii) image reversal, and (iii) direct-write, with all cases imaged using the same SPL equipment in the AFM mode. It is to be emphasized that SPL results presented here are not representative of the resolution capability of the technique, as atomic scale resolution was demonstrated before. ${ }^{9,23}$ For the sole purpose of applying LER metrology along with AFM imaging to SPL, the aforementioned image reversal process is chosen as a case study.

\section{LINE EDGE ROUGHNESS THEORY AND BACKGROUND}

LER is described as the roughness of random variations on a printed pattern. Similarly, LWR represents variations over the width of a patterned feature. ${ }^{24}$ For LER investigation, deviations from a straight edge are examined, while for LWR, it is required to examine fluctuations in the distance between two opposing edges. Several sources contribute to LER depending on the specific lithography technique. Among the leading sources of LER, one can mention LER of mask patterns in the case of hard mask use, variations in the exposure dose, photon flux variation (shot noise), nonuniformity in the chemical composition of the resist, and acid diffusion during chemical amplification, development, dynamic and static speckle. ${ }^{25}$
LER can be investigated using several tools such as SEM, AFM, and their special variations such as critical-dimension SEM (CD-SEM). CD-SEM and CD-AFM can quantify LER more precisely for small feature sizes, can be automated, and provide information about line wall profiles. Each method has its tradeoffs. For example, in SEM, charging of the resist pattern causes blurring of the image, especially along the edges. Although AFM provides a higher accuracy in this respect, it is a slower method compared to SEM. ${ }^{26}$ Under suitable conditions, AFM has a subnanometer spatial resolution approaching the atomic scale. ${ }^{27}$ The finite size of AFM tips is one of the biggest challenges in AFM metrology. Furthermore, conventional top-down AFM devices with common cone-shaped tips are not suitable to obtain information on sidewalls. For this reason, three-point tip and flared tip are proposed to access the sidewall profile. Sample tilt and rotation of the sample by $90^{\circ}$ are also proposed to collect sidewall information. A special variation of dual servo, flared tip AFM, called CD-AFM, can be used in sidewall profiling. ${ }^{28}$ However, CD-AFM has a larger tip diameter $(\sim 50 \mathrm{~nm})$ with a vertical edge radius of $20 \mathrm{~nm}$, making the measurement of high-frequency components of roughness harder. Additionally, the equipment's noise level is comparably high $(\sim 3-4 \mathrm{~nm})$, and CD-AFM is not capable to scan the space under $90 \mathrm{~nm}$ for dense features. ${ }^{29}$ Current limitations of metrology tools such as resolution, speed, and uncertainty necessitate a combination of multiple metrology tools for the metrology of next-generation semiconductor devices. ${ }^{30}$

LER can be calculated using either online or off-line image analysis. Online LER analysis is a real-time measurement technique that is automated, easier, and faster. In the online analysis, the number of the sampling points might be limited depending on the particular SEM setup and resist can be overexposed while imaging. Off-line analysis can be run with either SEM or AFM imaging and has the advantages of avoiding any overexposure of the resist pattern in SEM, the possibility of the highest single-pixel resolution, and the availability of spatial and fractal analysis. ${ }^{31}$ It was shown that such off-line analysis can provide the same level of accuracy in LER quantification as that of the online analysis. ${ }^{32}$

IRDS provides guidelines for the measurement of LER and LWR based on the standard definition of Semiconductor Equipment and Materials International according to which LER and LWR are calculated as $3 \sigma$ of residuals along $2-\mu \mathrm{m}$-long lines. ${ }^{3}$ Defined as the standard deviation of deviations from a straight line, $\sigma_{\mathrm{LER}}$ is given by

$$
\sigma_{\mathrm{LER}}=\sqrt{\frac{1}{N} \sum_{i=1}^{N}\left(x_{i}-\bar{x}\right)^{2}}
$$

with $\bar{x}=1 / N\left(\sum_{i=1}^{N} x_{i}\right)$, where $x_{i}$ is the local position of the edge point $i$ on the line, $\bar{x}$ is the average of the points on the line, and $N$ is the total number of points, as defined in the sketch in Fig. 1. LWR can also be calculated using the aforementioned method. The width of a line at the position $i, w_{i}$, can be calculated as follows:

$$
w_{i}=x_{i}^{R}-x_{i}^{L},
$$

with $x_{i}^{R}$ and $x_{i}^{L}$ defined in Fig. 1. 
a) Ideal Case

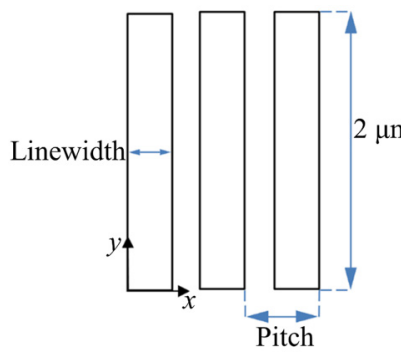

b)

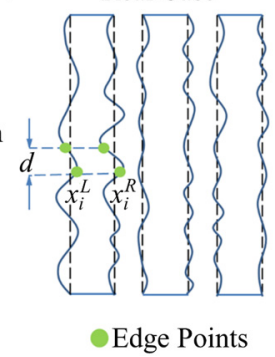

c)

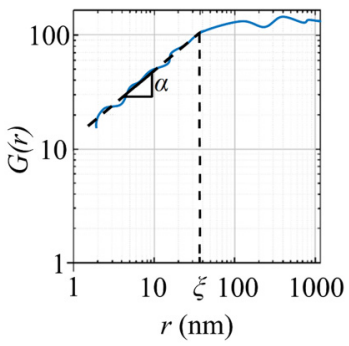

FIG. 1. Sketch of a series of resist lines in (a) ideal and (b) real cases with the illustration of the LER definition as a function of edge points with the distance $d$ between two consecutive points, and (c) representative HHCF of a line edge with correlation length, $\xi$, and roughness exponent, $\alpha$.
Standard deviation of linewidth, $\sigma_{\mathrm{LWR}}$, and the average width, $\bar{w}$, at the $i$ th interval can be found using Eqs. (3) and (4), respectively,

$$
\begin{gathered}
\sigma_{\mathrm{LWR}}=\sqrt{\frac{1}{N} \sum_{i=1}^{N}\left(w_{i}-\bar{w}\right)^{2}}, \\
\bar{w}=\frac{1}{N} \sum_{i=1}^{N} w_{i} .
\end{gathered}
$$

In addition to standard deviations of linewidth and line edge, spatial scaling and fractal analysis are necessary for the complete description of the edge profile. Frequency analysis provides the required correlation length, $\xi$, and fractal dimension, $D$, in this respect. These parameters can be obtained by using three different methods, namely, the height-height correlation function (HHCF), the Fourier transform, or the power spectral density. ${ }^{33}$ In this study, we utilized HHCF to find the spatial aspects of LER.

HHCF, represented by $G(r)$, describes the spatial distribution of roughness of an edge by studying the correlation between the distances of edge points from a linear fit of all edge points. ${ }^{32}$ $G(r)$ is defined at $r=m d$, with $m$ representing the degree of correlation and $d$ is the distance between two edge points along the $y$-axis [Eq. (5)],

$$
G(r)=\left[\frac{1}{N-m} \sum_{i=1}^{N-m}\left(\delta_{i+m}-\delta_{i}\right)^{2}\right]^{1 / 2},
$$

where $\delta_{i}$ is the distance of an edge point from the linear fit and $N$ is the total number of equidistant points along the $y$-axis. The linear portion of HHCF exhibits a power law behavior, whose slope defines the roughness exponent, $\alpha$, as shown in Fig. 1(c). $\alpha$ is, in turn, related to the fractal dimension, $D$. In HHCF, the power law behavior extends up to a certain point, beyond which $G(r)$ stabilizes. The distance at which HHCF assumes $\sqrt{1-1 / e}$ of its maximum value is defined as the correlation length, $\xi$, again depicted in Fig. 1(c).

\section{EXPERIMENTAL PROCEDURE}

The demonstration of the success of the line edge roughness software is carried out using current-controlled FE-SPL, a tip-based nanofabrication method relying on self-actuating and self-sensing active cantilevers. ${ }^{34}$ A scanning probe enables the user to image, align, pattern, and inspect a surface at the atomic scale. This versatile nature of the active scanning probes makes them an irreplaceable tool in many nanofabrication, positioning, overlay, and characterization applications. AFM images of the patterned features can be obtained directly after exposure using the same tool and cantilever. In the literature, direct fabrication of features smaller than $10 \mathrm{~nm}$ on molecular glass resist calixarene was reported using FE-SPL without development. ${ }^{35}$ Scaling the feature sizes down to the single-digit region allows one to utilize quantum-based effects such as Coulomb blockade and single-electron tunneling. ${ }^{36}$

While patterning, a bias-induced, Fowler-Nordheim-type electron emission takes place at the sharp tip. ${ }^{37}$ The resulting flux of low-energy electrons is regulated by a current feedback control in the SPL system and modulated by respective controller parameters. The resist can be either crosslinked or ablated, i.e., directly removed, depending on the applied exposure dose, a combination of speed and current, and applied bias voltage. ${ }^{38}$ While low exposure doses result in cross-linking of the resist (negative-tone patterning), higher exposure doses result in direct-write (positive-tone patterning). Similarly, positive-tone patterning can be achieved by image reversal.

The working principle of FE-SPL with two feedback control loops is demonstrated in Fig. 2(a). The setup utilizes thermally actuated, piezoresistive cantilevers for both lithography and metrology purposes. The use of such active cantilevers enables higher flexibility, higher speeds, and easier integration compared to the existing passive cantilever methods. ${ }^{39}$ The SPL platform consists of an approach system and a top scanner system, where the cantilever is mounted. The FE-SPL can pattern an area of up to $100 \times 100 \mathrm{~mm}^{2}$ and scan an area of $200 \times 200 \mu \mathrm{m}^{2}$ with an additional bottom stage enabling step-and-repeat operation. Both AFM and SPL are performed under room conditions keeping the temperature and relative humidity constant at $22 \pm 1{ }^{\circ} \mathrm{C}$ and $35 \pm 5 \%$, respectively, which are continuously monitored and recorded during patterning.

Among all other lithographic methods, FE-SPL provides the unique capability of scanning latent images of exposed features before development. Similarly, it allows one to observe any other local topography change in the resist induced by exposure. This observation facilitates the evaluation of the effect of development on the final shape of the structure. Observation of the latent image through AFM (Refs. 40 and 41) allows one to understand the contribution of process parameters to LER and linewidth. Generally, the height of the structures in latent images is on the order of $2 \mathrm{~nm}$ 
a)
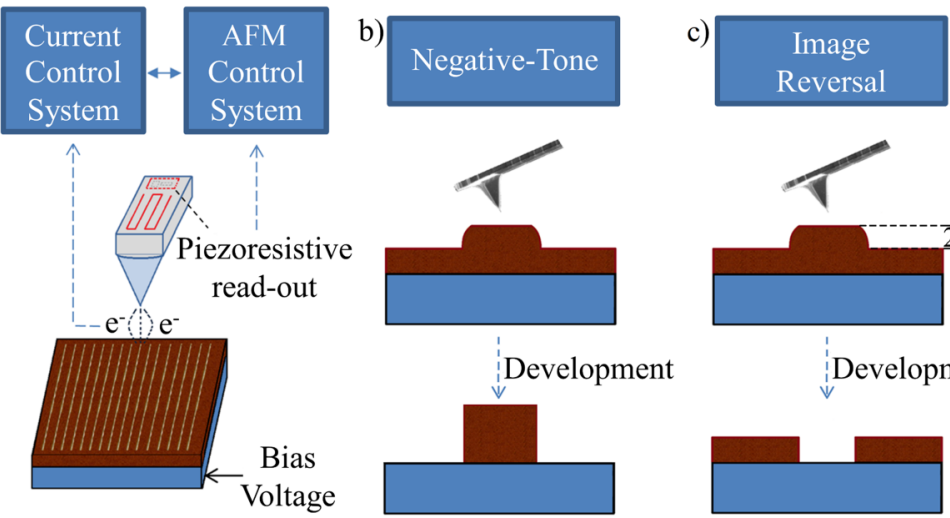

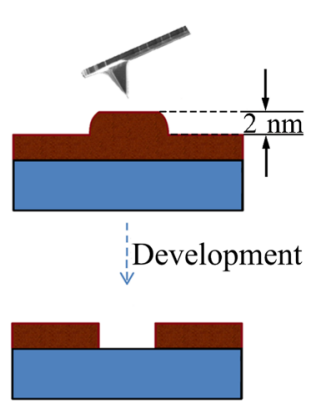

FIG. 2. (a) Working principle of current-controlled FE-SPL with two feedback loops utilizing thermally actuated piezoresistive cantilevers; (b) negative-tone patterning upon development in MIBK and (c) image reversal upon development in PMGI101A, both showing the latent image of about 2-nm height after exposure. making it very challenging to measure using techniques other than the noncontact AFM mode. When the patterned structures are exposed in negative tone and then developed in methyl isobutyl ketone (MIBK) solution, unexposed areas are removed, thereby yielding the resist profile shown in Fig. 2(b). If the latent features after negative-tone patterning are developed in the PMGI101A developer, an image reversal from negative-tone to positive-tone takes place as depicted in Fig. 2(c). In the present study, the latent image is directly obtained after exposure in the noncontact AFM mode of the same FE-SPL device.

For patterning purposes, a 10-nm-thick spin-coated calixarene molecular glass resist was used on an $\mathrm{Si}$ wafer with a resistivity of $1-15 \Omega \mathrm{cm}$. For each case of image reversal and direct-write, three exposure areas were utilized to study LER. In each exposure area, 10 lines with a nominal pitch of $200 \mathrm{~nm}$ were patterned with two different exposure doses of 25 and $200 \mathrm{nC} / \mathrm{cm}$ for negative tone and positive tone, respectively. The bias voltage was set to $40 \mathrm{~V}$ for a stable lithography. It is to be emphasized that this particular set of exposure doses and bias voltage values were chosen to keep linewidths at their minimum without compromising the stability of lithography. The linewidth of each feature is increased as a result of increasing exposure dose and bias voltage in positive-tone as well as in negative-tone patterning. ${ }^{18}$ After the exposure, the lines were scanned using the same SPL tip in the noncontact AFM mode. Later, the negative-tone sample was developed by using a PMGI101A developer for $5 \mathrm{~s}$ for image reversal, whereas no development was necessary in the case of direct-write (Fig. 3). After development, the same sample was scanned one more time to observe LER after image reversal. Special attention was paid to scanning the same area for overlapping latent image and image reversal.

Generally, the SPL tip is subject to wear and tear. Tips can be either broken completely or double-tip behavior can be observed after several uses, affecting the performance of lithography and imaging. Within this study, the SPL tip was characterized under SEM before patterning and imaging to ensure the sharpness of the tip as shown in Fig. 4. The tip-radius of curvature was approximately $15 \mathrm{~nm}$ for the presented tip, whereas diameters typically smaller than $10 \mathrm{~nm}$ are obtained. ${ }^{42}$ The height of the crystallographic Si tip is about $6 \mu \mathrm{m}$.

In line with the corresponding IRDS recommendation, imaging and image analysis were conducted by scanning lines with
$2 \mu \mathrm{m}$ length in a $2 \times 2 \mu \mathrm{m}^{2}$ window. Images were taken with the highest possible resolution of the TU-Ilmenau SPL setup, i.e., $1024 \times 1024 \mathrm{px}$, with a pixel size of $1.95 \mathrm{~nm}$.

We used the Image Processing toolbox of MATLAB with the Canny edge detection algorithm. The Canny algorithm is based on the calculation of the intensity gradients using the derivative of a Gaussian filter. The size and the standard deviation of this Gaussian filter are the two parameters that need to be carefully selected, as any improper selection might lead to inaccurate results. ${ }^{43}$ Hence, filter parameters should be optimized by observing the dependence of the correlation length on the standard deviation of the Gaussian filter. The signal threshold with high and low threshold parameters obtained by Otsu's method was applied to remove weak edges. ${ }^{8}$ All edges with an intensity below the lower threshold were disregarded, whereas those with an intensity above the high threshold were preserved. While detecting the edge points, there were some missing pixels along the line edge, which were

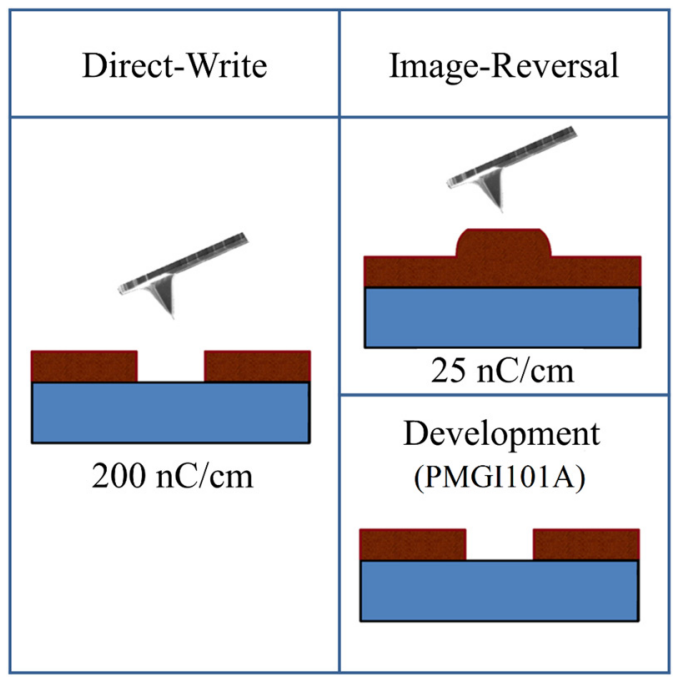

FIG. 3. Summary of the SPL exposures used in this study. Results of directwrite were compared to those of image reversal. 


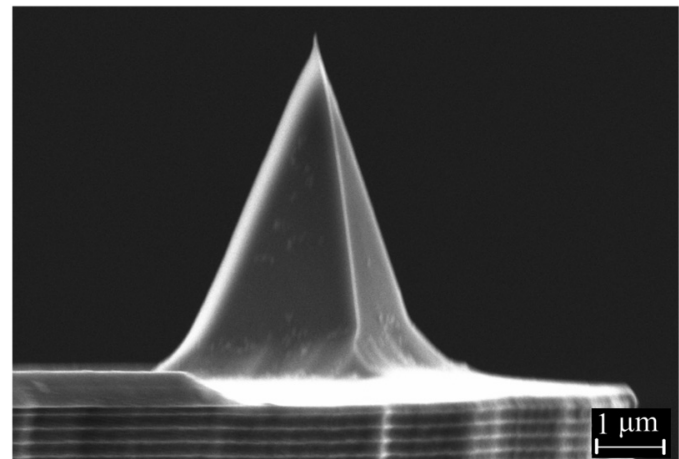

FIG. 4. SEM image of the active cantilever tip employed in FE-SPL before use.

replaced using a suitable interpolation by considering the continuity of the line. Spatial analysis was conducted by calculating HHCF of the lines on the image. The corresponding correlation length and roughness exponent were calculated from HHCF.

\section{RESULTS AND DISCUSSION}

AFM images collected after exposure were analyzed for latent image characterization. With a step height of about $2 \mathrm{~nm}$ for latent features, the resolution capability of FE-SPL tool in the
AFM mode was instrumental in in situ imaging-a challenging task for any other imaging approach. After development in PMGI101A, the same features were imaged again. This amounts to two images per case for image reversal characterization (second column in Fig. 3). On the contrary, since direct-write is a single-step process without development, one AFM image for each exposure was sufficient. With three areas exposed in each case, nine AFM images in total were studied for latent image, image reversal, and direct-write.

After edge point positions were detected, the corresponding LER, LWR, and spatial parameters were calculated using the formulation as laid out by Eqs. (1)-(5). Figures 5(a)-5(c) illustrate detected edge points superimposed on the actual representative AFM images in grayscale for latent image, image reversal, and direct-write, respectively. A close-up view with three lines is presented below each image. The corresponding HHCF for latent image, image reversal, and direct-write are depicted in Figs. 5(d)-5(f), respectively.

Roughness values and related correlation length, $\xi$, as well as the roughness exponent, $\alpha$, were calculated and averaged for each line in the representative latent image in Fig. 5(a) and reported in the first column of Table I with the corresponding METROLER software (Factilia, LLC) predictions listed in the second column. Fractilia claims the software can be used in the measurement of images obtained from different types of tools including CD-SEM and AFM. Although the inverse line-scan model is introduced to subtract SEM effects in LER measurements, a recent paper shows a)
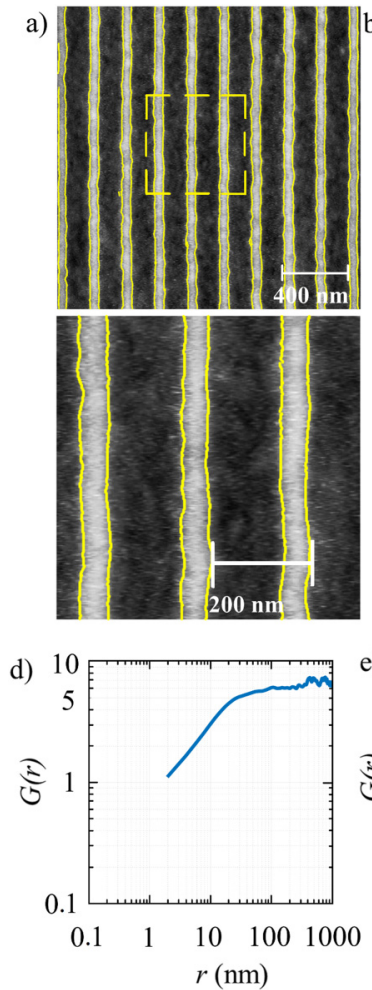
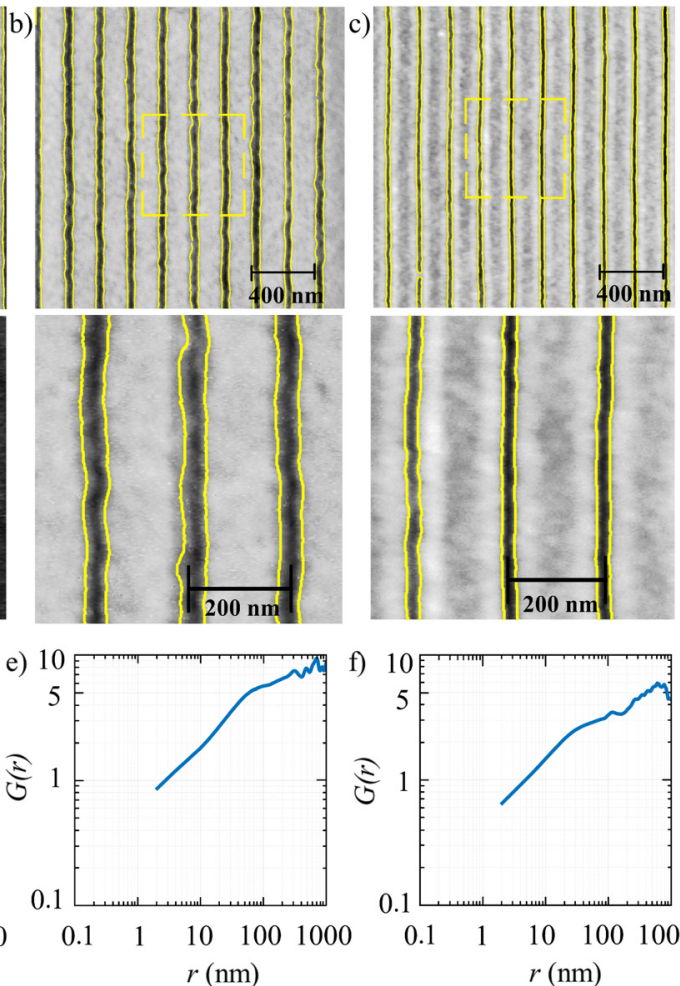

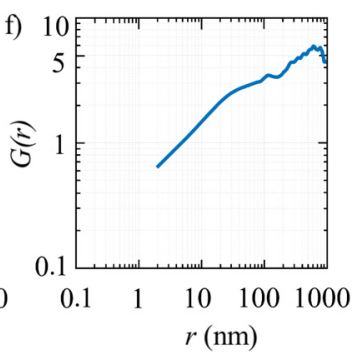

FIG. 5. Representative edge profiles extracted from AFM images of 10 lines for (a) negative-tone exposure (latent image), (b) image reversal, and (c) direct-write in FE-SPL with the insets showing close-up views and corresponding $\mathrm{HHCF}$ for (d) latent image, (e) image reversal, and (f) direct-write. 
TABLE I. Roughness parameters for latent image: Current analysis vs METROLER prediction obtained over 10 lines.

\begin{tabular}{lcc}
\hline \hline Parameter & Analysis & METROLER \\
\hline $3 \sigma_{\text {LER }}(\mathrm{nm})$ & $16.44 \pm 5.52$ & $17.90 \pm 1.72$ \\
$3 \sigma_{\text {LWR }}(\mathrm{nm})$ & $21.74 \pm 5.98$ & $21.42 \pm 3.23$ \\
$\xi(\mathrm{nm})$ & $30.33 \pm 5.96$ & $53.96 \pm 3.10$ \\
$\alpha$ & $0.69 \pm 0.02$ & $0.50 \pm 0.00$ \\
Linewidth $(\mathrm{nm})$ & $55.16 \pm 2.08$ & $79.2 \pm 0.96$ \\
Pitch $(\mathrm{nm})$ & $212.1 \pm 1.5$ & $211.7 \pm 1.0$ \\
\hline \hline
\end{tabular}

that unbiased measurements using the inverse line-scan model can be applied to both SEM and AFM images with similar accuracy. ${ }^{44}$ The results of Table I demonstrate that our analysis is capable of capturing similar LER, LWR, and fractal values with METROLER software with a maximum of $9 \%$ deviation in LER and with a much better agreement in LWR.

To compare the outcome of image reversal and direct-write as depicted in Fig. 3, images presented in Figs. 5(b) and 5(c) were analyzed. The results are summarized in Table II. Let us first compare the results of our analysis with METROLER predictions. It is to be noted that in the case of image reversal, linewidth and LWR are in fairly good agreement with a maximum deviation of about $20 \%$. The deviation in LER was reduced to below $8 \%$. In directwrite, the deviation in LER increased to about $30 \%$, while deviations in linewidth and LWR grew more significantly. How these deviations are improved with increased number of samples will be discussed later.

Furthermore, a comparison of image reversal and direct-write independent of the type of analysis used leads to the following observations: In all cases, LER and LWR associated with image reversal were found to be higher than those of direct-write. In both studies, we found average pitch values to be close to the nominal value of $200 \mathrm{~nm}$. Direct-write resulted in a smaller $\alpha$ compared to image reversal with high-frequency components contributing to the roughness to a larger extent. A larger correlation length in direct-write indicates a smoother edge profile with flat hills and valleys as shown in Fig. 5(c).

The above analysis related to both Tables I and II was carried out using 10 lines that fit in a single $2 \times 2 \mu \mathrm{m}^{2}$ scan area. As a final study, we analyzed three $2 \times 2 \mu \mathrm{m}^{2}$ images amounting to 30 lines, the results of which are summarized in Table III. As clearly seen from Table III, FE-SPL was again able to reproduce lines with a designated nominal pitch value of $200 \mathrm{~nm}$. The following observations were further noted:

(1) Increasing the number of lines did not cause any significant change in LER and LWR in the case of latent image. Our analysis led to the same LER of $16.44 \mathrm{~nm}$ with a slightly better spread obtained for 30 samples.

(2) For image reversal, the spread in LER became less with the average LER values remaining almost unchanged in our analysis. The corresponding deviation between our analysis and METROLER also decreased (about 6\%). The agreement for linewidth improved significantly with both methods yielding almost $50 \mathrm{~nm}$, although the deviation in LWR remained significant.

(3) For direct-write, the deviation for LER between the two methods as evident in Table II decreased significantly, although it remained almost unchanged for LWR. Similarly, the linewidth difference between our analysis and METROLER remained almost unchanged with both methods converging to constant values of 27 and $39 \mathrm{~nm}$, respectively.

It is to be emphasized once again that the particular set of exposure doses and bias voltages used for both image reversal and direct-write were picked up to keep linewidths at their minimum ensuring the stability of lithography. Apart from the number of samples, a general comparison of image reversal and direct-write leads to the following observations:

(1) In all cases, LER and LWR associated with image reversal were found to be higher than those obtained through direct-write, with a more significant reduction observed for LWR. Similarly, linewidth in image reversal was found to be higher than the linewidth of direct-write features.

(2) The significantly lower LER values in direct-write indicate that the patterning process through ablation is more effective for LER minimization compared to the development process associated with image reversal.

(3) Similarly, the spread in LER, which is consistently higher in image reversal, can be linked to the stochastic nature of the development process, where any process variations such as chemical composition and noise are amplified.

(4) In both studies, we found average pitch values to be close to the nominal value of $200 \mathrm{~nm}$. This indicates accuracy and precision of the exposure tool during lithography and imaging.

TABLE II. Comparison of roughness parameters obtained from image reversal and direct-write calculated over 10 lines.

\begin{tabular}{lrrrr}
\hline \hline & \multicolumn{2}{c}{ Image reversal } & \multicolumn{2}{c}{ Direct-write } \\
\cline { 2 - 3 } Parameter & Analysis & METROLER & Analysis & METROLER \\
\hline $3 \sigma_{\text {LER }}(\mathrm{nm})$ & $15.99 \pm 7.98$ & $14.75 \pm 1.87$ & $9.44 \pm 1.62$ & $12.25 \pm 1.05$ \\
$3 \sigma_{\text {LWR }}(\mathrm{nm})$ & $20.18 \pm 8.61$ & $17.19 \pm 2.57$ & $6.02 \pm 2.87$ & $12.65 \pm 1.25$ \\
$\xi(\mathrm{nm})$ & $35.79 \pm 6.82$ & $52.07 \pm 14.58$ & $48.34 \pm 7.13$ & $40.86 \pm 10.01$ \\
$\alpha$ & $0.70 \pm 0.02$ & $0.51 \pm 0.04$ & $0.66 \pm 0.02$ & $0.51 \pm 0.04$ \\
Linewidth $(\mathrm{nm})$ & $43.68 \pm 4.06$ & $52.1 \pm 3.10$ & $24.48 \pm 0.36$ & $39.7 \pm 1.30$ \\
Pitch $(\mathrm{nm})$ & $201.4 \pm 4.62$ & $201.2 \pm 1.70$ & $200.9 \pm 1.06$ & $200.3 \pm 0.44$ \\
\hline \hline
\end{tabular}


TABLE III. Roughness parameters for all exposure types obtained on 30 lines where our analyses are indicated in bold.

\begin{tabular}{lccr}
\hline \hline Parameter & Latent image & Image reversal & Direct-write \\
\hline $3 \sigma_{\text {LER }}(\mathrm{nm})$ & $\mathbf{1 6 . 4 4} \pm \mathbf{4 . 6 9}$ & $\mathbf{1 6 . 7 8} \pm \mathbf{5 . 8 1}$ & $\mathbf{1 0 . 9 5} \pm \mathbf{2 . 4 7}$ \\
$3 \sigma_{\text {LWR }}(\mathrm{nm})$ & $18.15 \pm 2.46$ & $17.89 \pm 1.62$ & $11.31 \pm 0.60$ \\
$\xi(\mathrm{nm})$ & $\mathbf{2 0 . 6 3} \pm \mathbf{5 . 6 4}$ & $\mathbf{1 6 . 5 6} \pm \mathbf{7 . 2 4}$ & $\mathbf{5 . 5 7} \pm \mathbf{2 . 9 2}$ \\
& $21.77 \pm 3.37$ & $22.23 \pm 2.70$ & $12.18 \pm 0.94$ \\
$\alpha$ & $\mathbf{2 7 . 6 8} \pm \mathbf{5 . 2 1}$ & $\mathbf{3 2 . 1 3} \pm \mathbf{4 . 2 7}$ & $\mathbf{3 6 . 3 5} \pm \mathbf{4 . 7 6}$ \\
& $21.22 \pm 2.98$ & $23.30 \pm 1.84$ & $29.71 \pm 3.46$ \\
Linewidth $(\mathrm{nm})$ & $\mathbf{5 6 . 8 3} \pm \mathbf{3 . 5 0}$ & $\mathbf{5 0 . 0 1} \pm \mathbf{4 . 0 5}$ & $\mathbf{2 6 . 8 1} \pm \mathbf{3 . 4 8}$ \\
& $50.80 \pm 2.20$ & $50.00 \pm 2.00$ & $39.00 \pm 1.20$ \\
Pitch $(\mathrm{nm})$ & $\mathbf{2 0 3 . 1} \pm \mathbf{1 0 . 4 7}$ & $\mathbf{2 0 1 . 2} \pm \mathbf{3 . 9 9}$ & $\mathbf{1 9 9 . 9} \pm \mathbf{2 . 3 8}$ \\
& $202.5 \pm 2.50$ & $200.5 \pm 1.20$ & $198.9 \pm 0.94$ \\
\hline \hline
\end{tabular}

(5) Direct-write resulted in smaller $\alpha$ compared to image reversal as indicated by high-frequency components contributing to the roughness to a larger extent. Larger correlation length in directwrite also indicates that edge profile this time is smoother with flat hills and valleys as discussed earlier.

This work presents the first of its kind by patterning and analyzing lines using the same FE-SPL setup by detecting edge points on latent image, image reversal, and direct-write. Image reversal of calixarene molecular glass resist from negative-tone to positivetone is studied for the first time in FE-SPL. A methodology of LER investigation is presented to compare image reversal with directwrite, where the lines were fabricated using single exposure doses for image reversal $(25 \mathrm{nC} / \mathrm{cm})$ and direct-write $(200 \mathrm{nC} / \mathrm{cm})$ by considering the linewidth of the final structures and the stability of the patterning. In the future, this study can be extended by studying the effect of different exposure doses on LER. For the SPL in the single-digit nanometer regime, different probe scanning and analysis algorithms should be studied with better modeling of tip-sample interaction to improve scanning speed and reduce image artifacts. ${ }^{45}$ Although the Canny Edge method was selected in this study because of its reliability to detect true weak edges under noisy conditions, more mature methods can be introduced with increased resolution requirements. A further comparison with CD-SEM or CD-AFM will also be studied to validate the metrology capability of the current FE-SPL setup.

\section{CONCLUSION}

In this work, we report LER as a result of image reversal and direct-write in current-controlled FE-SPL. Three exposure areas were patterned with 10 lines per each area using a 10 -nm-thick calixarene molecular glass resist on an Si wafer. The effect of the number of lines on the resulting feature size measurements is discussed by conducting the benchmarking study for 10 and 30 lines. Image reversal and direct-write were utilized using 25 and $200 \mathrm{nC} / \mathrm{cm}$ exposure doses, respectively. The sample prepared by negative-tone exposure $(25 \mathrm{nC} / \mathrm{cm})$ was developed by the PMGI101A developer for $5 \mathrm{~s}$ for image reversal from negative-tone to positive-tone. All AFM images were obtained in the noncontact AFM mode using the same FE-SPL setup used for patterning. Additionally, line edge roughness of the latent image was studied. With the height of the latent features on the order of $2 \mathrm{~nm}$, resolution capability of the setup proved to be adequate for in situ imaging. This observation of the latent image was also crucial for understanding the effect of process parameters to LER of final structures.

A MATLAB script was generated to calculate LER, LWR, linewidth, and pitch values as well as spatial parameters. The Canny edge detection algorithm was applied to find edge points. Otsu's method was utilized to find threshold values required for the algorithm. From HHCF, correlation length and roughness exponent for each line were found. For the verification of presented methodology, we compared the proposed analysis with a commercially available software, METROLER, for all cases. Both analyses were able to produce comparable results with the best overlap obtained in the case of image reversal.

In both studies on image reversal and direct-write, we found average pitch values to be close to the nominal value of $200 \mathrm{~nm}$, indicating the accuracy and precision of the exposure tool during lithography and imaging. The analysis also showed that for the particular set of exposure conditions, LER and LWR associated with image reversal are higher than those obtained through direct-write, with a more significant reduction observed for LWR. Similarly, linewidth in image reversal is larger. The significantly lower LER values in direct-write indicate that the patterning process through ablation is more effective for LER minimization compared to the development process of image reversal with its stochastic nature amplifying process variations such as chemical composition and noise.

Finally, the following set of findings are achieved through the benchmarking study:

(1) Latent image: The results were captured well despite the demanding metrology requirements. Increasing the number of lines did not cause any significant change in LER and LWR with an LER of $16.44 \mathrm{~nm}$ with a slightly better spread obtained for 30 samples.

(2) Image reversal: With increased number of samples, the spread in LER became less with the average LER values remaining almost unchanged in our analysis. The corresponding deviation between our analysis and METROLER also decreased to about $6 \%$. The agreement for linewidth improved significantly with both methods yielding almost $50 \mathrm{~nm}$, although the deviation in LWR remained significant.

(3) Direct-write: Although LWR exhibited a certain deviation between the two methods, the LER deviation decreased significantly with increased number of samples. Similarly, the difference in linewidth between our analysis and METROLER remained almost unchanged with both methods converging to constant values of 27 and $39 \mathrm{~nm}$, respectively.

While a close match was obtained for line edge roughness in directwrite, a more significant deviation in linewidth characterization remains to be addressed in further studies. This study can be extended by utilizing other edge detection techniques, observing 
the effect of exposure dose on image reversal and direct-write and including a comparison with other metrology tools including CD-AFM and CD-SEM.

\section{ACKNOWLEDGMENTS}

The authors would like to acknowledge Fractilia, LLC for providing unbiased roughness analysis. They thank Mahmut Bicer and Martin Hoffman for fruitful discussions.

\section{REFERENCES}

${ }^{1}$ C. A. Mack, IEEE Trans. Semicond. Manuf. 24, 202 (2011).

${ }^{2}$ H. N. Khan, D. A. Hounshell, and E. R. H. Fuchs, Nat. Electron. 1, 14 (2018).

3"Metrology," International Roadmap for Devices and Systems (IRDSTM) 2017 Edition, see https://irds.ieee.org/editions/2017/metrology (2019).

4"Lithography," International Roadmap for Devices and Systems (IRDSTM) 2017 Edition, see https://irds.ieee.org/editions/2017/lithography (2019).

$\mathbf{5}_{\text {nanoLERTM_Nanometrisis, see http://www.nanometrisis.com/ }}$ products-services/nanoler/ (2019).

${ }^{6}$ Fractilia Computational Metrology, see https://www.fractilia.com/http://www. nanometrisis.com/products-services/nanoler/ (2019).

${ }^{7}$ G. F. Lorusso, V. Rutigliani, F. Van Roey, and C. A. Mack, Microelectron. Eng. 190, 33 (2018).

${ }^{8}$ M. Fang, G. Yue, and Q. Yu, Int. Symp. Inf. Process. 2, 109 (2009).

9. W. Rangelow et al., Proc. SPIE 10584, 1058406 (2018).

${ }^{10}$ T. T. Gotszalk et al., J. Vac. Sci. Technol. B 37, 030803 (2019).

${ }^{11}$ R. Garcia, A. W. Knoll, and E. Riedo, Nat. Nanotechnol. 9, 577 (2014).

${ }^{12}$ S. Lenk, M. Kaestner, C. Lenk, Y. Krivoshapkina, and I. W. Rangelow, 2017 30th International Vacuum Nanoelectronics Conference (IVNC 2017), 10-14 July 2017 (IEEE, Regensburg, Germany), p. 108.

${ }^{13}$ I. W. Rangelow et al., J. Vac. Sci. Technol. B 34, 06K202 (2016).

${ }^{14}$ M. Kaestner, M. Hofer, and I. W. Rangelow, Proc. SPIE 8680, 868019 (2013).

${ }^{15}$ C. Lenk et al., J. Vac. Sci. Technol. B 37, 021603 (2019).

${ }^{16}$ J. M. Shaw, J. D. Gelorme, N. C. LaBianca, W. E. Conley, and S. J. Holmes, IBM J. Res. Dev. 41, 81 (1997).

17. Fujita, Y. Ohnishi, Y. Ochiai, E. Nomura, and S. Matsui, J. Vac. Sci. Technol. B 14, 4272 (1996).

${ }^{18}$ M. Kaestner and I. W. Rangelow, Microelectron. Eng. 97, 96 (2012).
${ }^{19}$ L. L. Cheong, P. Paul, F. Holzner, M. Despont, D. J. Coady, J. L. Hedrick, R. Allen, A. W. Knoll, and U. Duerig, Nano Lett. 13, 4485 (2013).

${ }^{20}$ A. Saeki, T. Kozawa, S. Tagawa, H. B. Cao, H. Deng, and M. J. Leeson, J. Micro/Nanolithogr. MEMS MOEMS 6, 043004 (2007).

${ }^{21} \mathrm{~J}$. T. Woodward et al., Proc. SPIE 6923, 69232B (2008).

${ }^{22}$ M. Ishida, K. Kobayashi, J. I. Fujita, Y. Ochiai, H. Yamamoto, and S. Tono, Jpn. J. Appl. Phys. Part 1 41, 4228 (2002).

${ }^{23}$ I. W. Rangelow et al., Proc. SPIE 10456, 1045621 (2018).

${ }^{24}$ C. A. Mack, Field Guide to Optical Lithography (SPIE, Bellingham, 2006).

${ }^{25}$ T. Sandstrom and C. Rydberg, Proc. SPIE 6520, 65200X (2007).

${ }^{26}$ C. Nelson, S. C. Palmateer, A. R. Forte, and T. M. Lyszczarz, J. Vac. Sci. Technol. B 17, 2488 (1999).

${ }^{27}$ N. G. Orji, T. V. Vorburger, J. Fu, R. G. Dixson, C. V. Nguyen, and J. Raja, Meas. Sci. Technol. 16, 2147 (2005).

${ }^{28}$ N. G. Orji, M. I. Sanchez, J. Raja, and T. V. Vorburger, Nanoscience and Technolology (Springer, Berlin, 2014), pp. 277-301.

${ }^{29}$ M. Fouchier, E. Pargon, and B. Bardet, J. Appl. Phys. 113, 104903 (2013).

${ }^{30} \mathrm{~N}$. G. Orji et al., Nat. Electron. 1, 532 (2018).

${ }^{31}$ G. P. Patsis, V. Constantoudis, A. Tserepi, and E. Gogolides, J. Vac. Sci. Technol. B 21, 1008 (2003).

${ }^{32}$ V. Constantoudis, G. P. Patsis, L. H. A. Leunissen, and E. Gogolides, J. Vac. Sci. Technol. B 22, 1974 (2004).

${ }^{33}$ V. Constantoudis, G. P. Patsis, A. Tserepi, and E. Gogolides, J. Vac. Sci. Technol. B 21, 1019 (2003).

${ }^{34}$ M. Kaestner et al., Proc. SPIE 9423, 94230E (2015).

${ }^{35}$ M. Kaestner and I. W. Rangelow, J. Vac. Sci. Technol. B 29, 06 FD02 (2011).

${ }^{36}$ Z. Durrani, M. Jones, M. Kaestner, M. Hofer, E. Guliyev, A. Ahmad, T. Ivanov, J.-P. Zoellner, and I. W. Rangelow, Proc. SPIE 8680, 868017 (2013).

${ }^{37}$ M. Kaestner et al., Proc. SPIE 9049, 90490C (2014).

${ }^{38}$ Y. Krivoshapkina, M. Kaestner, C. Lenk, S. Lenk, and I. W. Rangelow, Microelectron. Eng. 177, 78 (2017).

${ }^{39}$ I. W. Rangelow et al., J. Vac. Sci. Technol. B 35, 06G101 (2017).

${ }^{40}$ L. E. Ocola, D. S. Fryer, G. Reynolds, A. Krasnoperova, and F. Cerrina, Appl. Phys. Lett. 68, 717 (1995).

${ }^{41}$ D. L. Olynick, J. A. Liddle, A. V. Tivanski, M. K. Gilles, T. Tyliszczak, F. Salmassi, K. Liang, and S. R. Leone, J. Vac. Sci. Technol. B 24, 3048 (2006).

${ }^{42}$ C. Lenk et al., J. Vac. Sci. Technol. B 36, 06JL03 (2018).

${ }^{43}$ C. A. Mack and B. D. Bunday, Proc. SPIE 10145, 101451R (2017).

${ }^{44}$ G. F. Lorusso et al., J. Micro/Nanolithogr. MEMS MOEMS 17, 041009 (2018).

${ }^{45}$ H.-C. Liu, G. A. Dahlen, and J. R. Osborne, Applied Scanning Probe Methods VIII (Springer, Berlin, 2008), pp. 31-75. 